\title{
Competition between ethanol-induced reward and aversion in place conditioning
}

\author{
CHRISTOPHER L. CUNNINGHAM, RACHEL SMITH, and CARRIE MCMULLIN \\ Oregon Health \& Science University, Portland, Oregon
}

\begin{abstract}
Previous place conditioning studies in mice have shown that injection of ethanol immediately before a conditioned stimulus (CS+) produces conditioned preference, whereas injection of ethanol immediately after $\mathrm{CS}+$ produces conditioned aversion. In the present experiments, we examined the learning that occurs when ethanol is injected in "ambiguous" procedures that provide the opportunity for both types of conditioning. When ethanol was given midway through the CS (Experiments 1 and 2) or both before and after the CS (Experiment 3), the direction of place conditioning was the same as when mice were exposed only to whichever contingency occurred first (a primacy effect). That is, injection of ethanol in the middle of the CS conditioned aversion, whereas injection both before and after the CS conditioned preference. Because these results support the idea that ethanol elicits both aversive and rewarding effects, they are most consistent with conditioning theories that conceptualize unconditioned stimuli (USs) as events that can activate multiple representational components.
\end{abstract}

Events that serve as unconditioned stimuli (USs) in conventional Pavlovian conditioning procedures are generally treated as if they have a univalent motivational effect. That is, some events (e.g., food, water, access to a sexually receptive conspecific) are considered to be motivationally positive (appetitive or rewarding) on the basis of their common ability to support approach and contact behaviors, whereas other events (e.g., electric shock, illness) are considered to be motivationally negative (aversive) on the basis of their ability to produce escape and avoidance behaviors. Although the motivational categorization of a particular event may differ across individuals (e.g., as a function of subject variables such as age, strain, or sex) or may change as a function of experience, traditional descriptions of Pavlovian conditioning have typically assumed that the US on any given trial is either appetitive or aversive but not both (e.g., Rescorla \& Solomon, 1967).

However, conditioning studies involving abused drugs have raised the interesting possibility that drug USs may, in some cases, elicit both rewarding and aversive effects, perhaps even at the same time. A prototypical example of this possibility is demonstrated in studies showing that the same injection of an abused drug such as amphetamine can produce both conditioned aversion to the taste of a paired flavor solution and conditioned preference for the spatial location in which the flavored solution is consumed (e.g., Reicher \& Holman, 1977). A common interpretation of this finding is that the drug experience is indeed both rewarding and aversive, but that these motivationally op-

This research was supported by Grants AA07702 and AA07468 from NIH-NIAAA. Correspondence should be addressed to C. L. Cunningham, Department of Behavioral Neuroscience L470, Oregon Health \& Science University, 3181 SW Sam Jackson Park Road, Portland, OR 97239-3098(e-mail: cunningh@ohsu.edu). posite unconditionedeffects are selectively associated with exteroceptive and interoceptive conditioned stimuli (CSs), respectively (Reicher \& Holman, 1977).

Subsequent studies have extended this analysis to include the possibility that the same exteroceptive CS may become associated with either the positive or the negative motivational effects of a drug, depending on the temporal relationship between the CS and the drug US. For example, in a series of studies conducted in rats, doses of nicotine and amphetamine that produce conditioned place preference when injected immediately before exposure to the conditioning environment were found to produce conditioned place aversion when injected after the animal was removed from the environment (Fudala \& Iwamoto, 1987, 1990). Although the impact of these findings is weakened somewhat by reports that pre-CS injection of nicotine will sometimes condition place aversion (Jorenby, Steinpreis, Sherman, \& Baker, 1990; Risinger \& Oakes, 1995), a very similar phenomenon has recently been reported in a series of ethanol place conditioning studies in mice (Cunningham \& Henderson, 2000; Cunningham, Henderson, \& Bormann, 1998; Cunningham, Okorn, \& Howard, 1997; Cunningham, Tull, Rindal, \& Meyer, 2002). That is, post-CS injection of ethanol produced conditioned place aversion in mice, even though pre-CS injection of the same dose was consistently found to produce conditioned place preference. The place aversion induced by post-CS ethanol injection was positively related to ethanol dose, inversely related to conditioning trial duration, reduced by extinction of the CS, and reduced by home cage preexposure injections of ethanol (Cunningham\& Henderson, 2000; Cunningham, Tull, et al., 2002).

Cunningham and colleagues have suggested that the place aversion conditioned by post-CS ethanol injection reflects an aversive motivationaleffect that is mediated in- 
dependently of the rewarding effect that presumably underlies the conditioned place preference induced by pre-CS injection. More specifically, they have proposed that ethanol injection produces a short-lived aversive effect that reflects the novelty of the relatively rapid transition from the sober state to the intoxicated state. This interpretation is strongly supported by studies showing that familiarity with ethanol reduces or eliminates conditioned place aversion but does not affect conditioned place preference (Cunningham, Tull, et al., 2002).

Given the possibility that ethanol and other abused drugs elicit both rewarding and aversive effects and that either effect may become associated with exteroceptive cues, it is of interest to consider what happens when the temporal arrangement between the CS and the US puts these motivational effects into competition with each other. We addressed this issue in the present study by administering the ethanol US in two different temporal arrangements that could be considered ambiguous. In one case (Figure 1A), ethanol was injected halfway through a 10 -min conditioning trial (Experiments 1 and 2). When viewed as two consecutive 5-min conditioning trials, the first half of the trial contained a contingency (ethanol after CS) that has been demonstrated many times to produce a reliable conditioned place aversion in mice (Cunningham \& Henderson, 2000; Cunningham et al., 1998; Cunningham et al., 1997; Cunningham, Tull, et al., 2002), whereas the second half of the trial provided a contingency (ethanol before CS) that has repeatedly been shown to produce a robust conditioned place preference (e.g., Cunningham, 1995; Cunningham, Dickinson, \& Okorn, 1995; Cunningham et al., 1998; Cunningham et al., 1997; Cunningham, Tull, et al., 2002). In the other case (Figure 1B), the experimental mice received two identical ethanol injections on each $\mathrm{CS}+$ trial, one immediately before CS exposure and an-
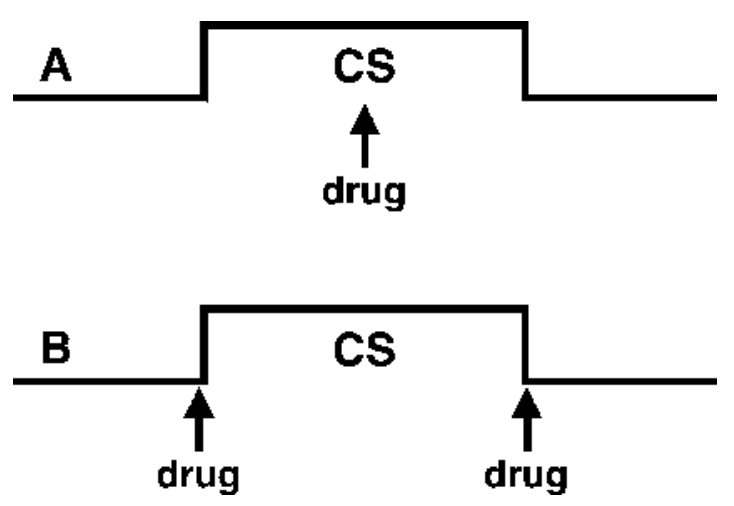

Figure 1. Diagrammatic representations of "ambiguous" place conditioning procedures used in Experiments 1 and 2 (panel A) and in Experiment 3 (panel B). Panel A: The drug is injected midway through a 10 -min conditioned stimulus $(C S+)$ conditioning trial, allowing the CS both to precede the onset of ethanol's effects and to overlap the first $5 \mathrm{~min}$ of intoxication. Panel B: The drug is injected before and again after exposure to the 5-min CS+, allowing the CS to overlap the first 5 min of intoxication and to precede the onset of drug effects produced by the second injection. other immediately afterward (Experiment 3 ). When given to independent groups, these injections would be expected to produce conditioned place preference and conditioned place aversion, respectively (Cunninghamet al., 1997). The question of interest in both cases was whether the hypothesized bivalent effects of ethanol would simply offset each other or whether one effect would be a greater than the other.

\section{GENERAL METHOD}

\section{Subjects}

Naive adult male mice (DBA/2J) were shipped from the Jackson Laboratory (Bar Harbor, ME) at 6 weeks of age (Experiment 1, $n=$ 72; Experiment 2, $n=48$; Experiment 3, $n=93$ ). They were housed in groups of three or four in polycarbonate cages with cob bedding in a Thoren rack and were allowed to adapt to the colony for about 2 weeks before testing. The colony was maintained at $21^{\circ} \pm 1^{\circ} \mathrm{C}$ with a normal 12:12-h light:dark cycle (lights on at 7 a.m.). Testing occurred during the light cycle. Food and water were available at all times in the home cage. The NIH principles of laboratory animal care were followed in conducting these experiments.

\section{Apparatus}

Place conditioning and preference testing were conducted in 12 aluminum and acrylic chambers $(30 \times 15 \times 15 \mathrm{~cm})$ contained in separate ventilated sound- and light-attenuating enclosures (Coulbourn Instruments Model E10-20). Side position was detected by six sets of infrared light sources and photodetectors mounted at equal intervals $2.2 \mathrm{~cm}$ above the floor along the length of each chamber. In order to examine possible effects of test session activity levels on the expression of place conditioning (see Cunningham, 1995), occlusion of these infrared detectors was also used to measure activity (each beam break registered one activity count). The data were automatically recorded by a microcomputer. CSs were provided by the tactile cues of interchangeable floor halves placed beneath each chamber. The grid floor was constructed from $2.3-\mathrm{mm}$ stainless steel rods mounted $6.4 \mathrm{~mm}$ apart in acrylic rails. The hole floor consisted of stainless steel sheet metal (16 gauge) perforated with $6.4-\mathrm{mm}$ round holes on $9.5-\mathrm{mm}$ staggered centers. This combination of floor stimuli was selected on the basis of many previous studies indicating that saline-treated mice spend about half their time on each floor type during choice tests (e.g., Cunningham, 1995; Cunningham, Niehus, Malott, \& Prather, 1992; Cunningham et al., 1997). The inside of the chamber and the floors were wiped with a damp sponge and the litter paper beneath the floors was changed after each animal.

\section{EXPERIMENT 1}

The experimental mice were injected with ethanol at the midpoint of exposure to the CS (Figure 1A). Thus, the ethanol US was presented both before and after a period of CS exposure on each trial. Depending on assumptions about the relative strengths of the hypothesized opposing motivational effects and the importance of temporal sequencing during these back-to-back trials, one can make several different predictions. If the two effects differ in strength and temporal sequencing is not important, the behavioral outcome will presumably reflect whichever motivational effect is stronger. In contrast, if the opposing motivational effects are of equal strength, they will presumably cancel each other out, resulting in no place conditioning. However, if temporal sequence is critical, the CS might form an association primarily or exclusively 
with ethanol's aversive effect (a primacy effect) or with its rewarding effect (a recency effect). To assess the impact of the middle-injection procedure, the experimental group (middle group) was compared with groups that always received the same dose of ethanol immediately before (before group) or after (after group) exposure to the 10-min CS.

\section{Method}

This experiment included three phases: habituation (one 10-min session), conditioning (eight 10-min sessions), and a place preference test (one 60-min session). On the habituation day, the mice received an intraperitoneal (i.p.) saline injection followed by $10 \mathrm{~min}$ of exposure to a smooth paper floor in the apparatus. The mice were then randomly assigned to one of three treatment groups: before, middle, or after ( $n=24$ per group). Within each of these groups, the mice were randomly assigned to either the grid + or grid - conditioning subgroup ( $n=12$ per subgroup) and were exposed to a series of Pavlovian conditioning trials beginning $24 \mathrm{~h}$ after the habituation session. Each conditioning trial lasted $10 \mathrm{~min}$, and the mice had access to the entire chamber, which had the same floor type on both sides. On alternate days, the mice in the grid + subgroups received an i.p. injection of ethanol ( $2 \mathrm{~g} / \mathrm{kg}, 20 \% \mathrm{v} / \mathrm{v}$ in saline) paired with exposure to the grid floor and an injection of saline paired with exposure to the hole floor. These contingencies were reversed for the mice in the grid - subgroups. The mice in the before group received their ethanol injections immediately before placement on the assigned floor, a procedure that was expected to condition preference for the ethanol-paired floor. The mice in the after group received the ethanol injection immediately after removal from the floor, a procedure that was expected to condition aversion to that floor. However, the mice in the middle group initially received $5 \mathrm{~min}$ of exposure to the assigned floor without the drug. They were then removed, injected with ethanol (CS + trials) or saline (CS - trials), and quickly returned to the same floor for the remainder of the 10min trial.

For all groups, the four conditioning trials were conducted over an 8-day period, and the order of CS + and CS - trials was counterbalanced within each conditioning subgroup. A 2-day break occurred between the fourth and fifth conditioning sessions. On the day after the last conditioning trial, all the mice received a 60 -min floor preference test. Each half of the floor had a different texture (grid vs. hole), and floor position (left vs. right) was counterbalanced within each conditioning subgroup. The primary dependent variable was the amount of time spent on the grid floor during the test (expressed as seconds/minute on the grid floor).

\section{Results}

Four mice in the after group were removed from the experiment due to procedural errors, leaving a group size of 20 . Both of the other groups contained 24 mice.

In this experimental design, a comparison of the time spent on the grid floor by the counterbalanced grid + and grid - subgroups within each treatment group provides evidence of place conditioning(Cunningham,1993). Greater time spent on the grid floor by grid + subgroups than by grid- subgroups indicates the development of conditioned place preference, whereas the reverse indicates development of conditioned place aversion (Cunningham et al., 1997). As is shown in Figure 2, the before group displayed conditioned place preference, whereas the middle and after groups showed similar levels of conditioned place aversion. These observations were supported by a two- way analysis of variance (ANOVA; group $\times$ conditioning subgroup) that yielded a significant main effect of conditioning subgroup $[F(1,62)=22.2, p<.001]$ and a group $\times$ conditioning subgroup interaction $[F(2,62)=29.9, p<$ $.001]$. Follow-up comparisons confirmed that the difference between the grid + and the grid- subgroups was significant within each of the main treatment groups (Bonferronicorrected $p s<.002$ ).

The before group showed a higher level of test session activity (mean $\pm S E M=36.3 \pm 1.2$ counts $/ \mathrm{min}$ ) than did either the middle $(28.9 \pm 1.3)$ or the after $(26.6 \pm 1.4)$ group [groups effect, $F(2,65)=15.5, p<.0001]$. Follow-up tests confirmed that the before group was significantly more active than either of the other two groups (Bonferronicorrected $p<.001$ ), which did not differ.

\section{Discussion}

As in previous studies, pre-CS injection of ethanol produced conditioned place preference (before group), and post-CS injection produced conditioned place aversion (after group; e.g., Cunningham et al., 1997). The novel finding is that midtrial injection of ethanol (middle group) produced a conditioned place aversion that was indistinguishable from the aversion conditioned by post-CS injection. Thus, despite receiving a 5-min overlap of the CS with the onset of ethanol intoxication on each trial (a procedure that normally produces a robust conditioned place preference), the mice in the middle group behaved as though they had been exposed only to post-CS injections of ethanol. This outcome supports the idea that learning in

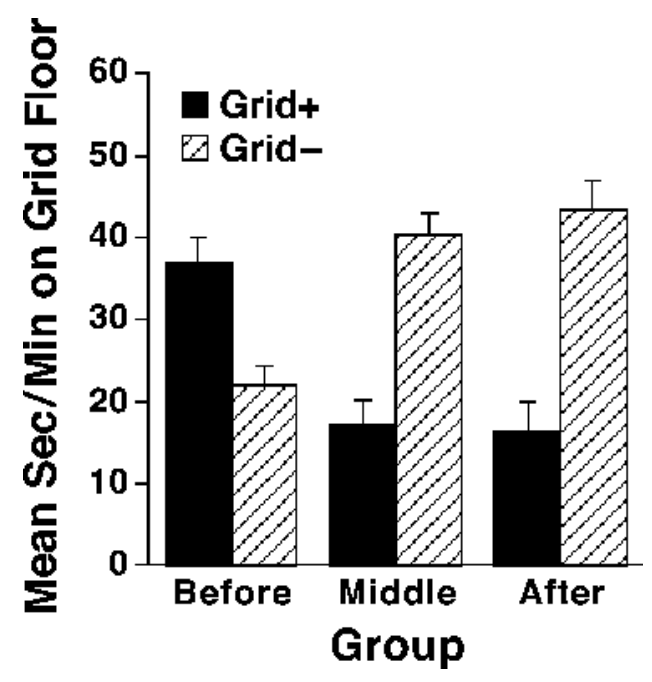

Figure 2. Mean seconds per minute $(+S E M)$ spent on the grid floor during the 60-min floor preference test session in Experiment 1 . During the conditioning phase, the mice had been given ethanol ( $2-\mathrm{g} / \mathrm{kg})$ injections immediately before (before group), midway through (middle group), or immediately after (after group) a 10-min exposure to the conditioned stimulus (CS+) floor. Grid + and grid - refer to the counterbalanced conditioning subgroups that received the grid floor paired or unpaired with ethanol injection, respectively $(n=9-12$ per subgroup $)$. 
this situation is governed by a primacy rule; that is, the first contingency experienced by the mouse (i.e., ethanol after CS) takes precedence in determining its subsequent behavior. However, this interpretation must be tempered by the fact that only one specific midtrial temporal arrangement of the CS and ethanol was examined. It is possible, for example, that a different temporal delay or different proportions of CS exposure before and after the midtrial ethanol injection could produce a weaker aversion than a post-CS injection would, or that it could even produce a conditioned place preference.

The finding of greater test session activity in the before group than in the after group is quite consistent with previous findings (see, e.g., Cunningham et al., 1998; Cunningham et al., 1997). Moreover, the similarity between the test activities of the middle and after groups provides further support for the observation that the middle group behaved as if it had been exposed to post-CS injections of ethanol. However, group differences in test session activity do not offer an alternative interpretation of the group differences in place conditioning. On the basis of previous data suggesting an inverse relationship between test session activity levels and strength of conditioned place preference (e.g., Cunningham, 1995), one might have predicted that the reduced level of activity in the middle (or after) group would be associated with an enhanced expression of conditioned preference. However, the literature offers no reason why reduced activity, per se, would produce a reversal in the direction of place conditioning.

It should be noted that this is not the first report of conditioned place aversion produced by midtrial drug exposure. Wall, Hinson, Schmidt, Johnston, and Streather (1990) reported that injection of amphetamine $15 \mathrm{~min}$ after the onset of a 45-min CS exposure produced conditioned place aversion in rats. Like Fudala and Iwamoto (1990), Wall et al. also found that post-CS injection of amphetamine produced conditioned place aversion in rats. However, in contrast to Fudala and Iwamoto and others (e.g., Reicher \& Holman, 1977), these investigators were not able to condition a place preference when amphetamine was injected immediately before CS exposure. Thus, their midtrial injection procedure cannot be considered ambiguous in the sense of putting amphetamine's bivalent motivational effects into competition. In fact, in trying to reconcile their findings with the literature, Wall et al. suggested that amphetamine's motivational effects may vary as a function of number of injections, with positive effects occurring after a small number of injections and negative effects occurring after many injections. That explanation, however, cannot be applied to the present situation, because the mice that had received the same total number of injections showed either conditioned place preference or aversion, depending on the temporal relationshipbetween the CS and ethanol injection.

In another place conditioning study that examined midtrial drug exposure, Bardo and Neisewander (1986) found that delaying intravenous infusion of morphine for 15 or 25 min after the onset of a 30-min CS eliminated the conditioned place preference that was normally produced in rats when the infusion coincided with CS onset. Aside from the obvious differences in drug and route of administration, their failure to observe conditioned place aversion with midtrial infusion might have been due to the use of only one conditioning trial as well as a "biased" procedure that reduced their ability to detect conditioned aversion (i.e., morphine was consistently paired with a white compartment that was normally avoided by no-drug control subjects).

\section{EXPERIMENT 2}

The outcome of Experiment 1 suggested that ethanol's aversive effect might have completely overpowered its rewarding effect in the mid-CS injection procedure. Experiment 2 was designed to see whether CS-ethanol overlap had produced any association with ethanol's rewarding effect. The strategy used to address this issue was based on a recent series of studies showing that two different forms of ethanol preexposure interfere with ethanol-induced conditioned place aversion but have no effect on conditioned place preference (Cunningham, Tull, et al., 2002). More specifically, these studies showed that a series of four home cage ethanol injections given during the week before place conditioning (distal preexposure) or a home cage ethanol injection given $1 \mathrm{~h}$ before each CS + trial (proximal preexposure) prevented the development of conditioned place aversion but did not alter conditioned place preference. Thus, the experimental mice in Experiment 2 received a combination of distal and proximal ethanol preexposures intended to reduce or eliminate conditioning of ethanol's aversive effects in the mid-CS injection procedure. If animals normally form independent associations between the CS and ethanol's rewarding and aversive effects in this procedure, the preexposure manipulation might be expected to unmask a conditioned place preference by selectively eliminating the conditioned aversion. However, if the mid-CS temporal arrangement actually prevents association with the rewarding effect, ethanolpreexposed mice would be expected to show no evidence of conditioning in either direction. To control for nonspecific effects of repeated handling and injection during preexposure, the control mice were injected with saline.

\section{Procedure}

There were three phases in this experiment: distal ethanol preexposure (four home cage injections), conditioning (eight 10-min sessions), and a place preference test (one 60-min session). During the distal preexposure phase, the mice were randomly assigned to one of two preexposure treatment groups (saline or ethanol; $n=24$ per group) that received a series of four i.p. injections of the assigned drug in the home cage at 48 -h intervals over the 8 days before conditioning. The ethanol dose was $2 \mathrm{~g} / \mathrm{kg}(20 \% \mathrm{v} / \mathrm{v})$.

Place conditioning began $48 \mathrm{~h}$ after the last distal preexposure injection. The mice within each preexposure group were randomly assigned to the grid + and grid - conditioning subgroups $(n=12$ per subgroup) that received a series of $\mathrm{CS}+$ and $\mathrm{CS}-$ conditioning trials (four of each type) identical to those received by the middle group in Experiment 1. However, the mice in the ethanol preexposure group also received proximal preexposure injections of ethanol $(2 \mathrm{~g} / \mathrm{kg}) 1 \mathrm{~h}$ before each $\mathrm{CS}+$ trial. These injections were expected, like distal preexposure, to interfere with the development of conditioned place aversion but not conditioned place preference (Cunningham, Tull, et al., 2002). The mice in the saline preexposure 
group received a proximal injection of saline $1 \mathrm{~h}$ before each of their $\mathrm{CS}+$ trials, and both groups received a proximal saline injection $1 \mathrm{~h}$ before each CS - trial. The experiment concluded with a $60-\mathrm{min}$ floor preference test as in the previous experiment.

\section{Results}

The saline preexposure group developed conditioned place aversion, whereas the ethanol preexposure group did not show either conditioned place aversion or preference (Figure 3). A two-way ANOVA (preexposure group $\times$ conditioning subgroup) revealed a significant main effect of conditioning subgroup $[F(1,44)=9.3, p<.01]$ and a significant interaction between these two factors $[F(1,44)=$ $5.3, p<.05]$. Follow-up comparisons showed that the difference between grid + and grid - was significant for the saline preexposure group (Bonferroni-corrected $p<.001$ ) but not for the ethanol preexposure group.

The ethanol preexposure group had significantly higher test activity levels $(29.9 \pm 0.9)$ than did the saline preexposure group $[25.5 \pm 0.9 ; F(1,46)=7.1, p=.01]$.

\section{Discussion}

As in recent studies involving post-CS ethanol injections (Cunningham, Tull, et al., 2002), ethanol preexposure prevented the development of conditioned place aversion in the mice given mid-CS injections of ethanol. The fact that the ethanol-preexposed mice did not express a conditioned place preference, which is known to be insensitive to ethanol preexposure, suggests that the mid-CS injection procedure does not normally establish an association between the CS and ethanol's rewarding effect.

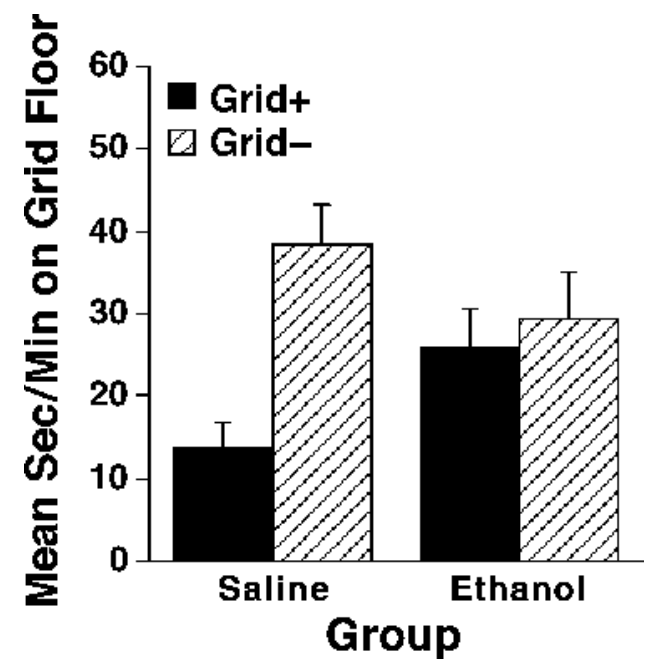

Figure 3. Mean seconds per minute $(+S E M)$ spent on the grid floor during the 60-min floor preference test session in Experiment 2 . Both the ethanol and saline groups were exposed to the conditioning procedure used for the middle group in Experiment 1 . The ethanol group received both distal and proximal preexposure injections of ethanol in the home cage (see the text), whereas the saline group received preexposure injections of saline. Grid+ and grid - refer to the counterbalanced conditioning subgroups that received the grid floor paired or unpaired with ethanol injection, respectively ( $n=12$ per subgroup).
Rather, this outcome is consistent with the suggestion that a mid-CS ethanol injection induces an association only with ethanol's aversive effect.

One possible interpretation of the higher test session activity observed in the ethanol-preexposedmice is that preexposure interfered with whatever process was responsible for the reduced levels of test activity seen in both the middle and after groups in Experiment 1 (e.g., interference with conditioning of activity suppression based on anticipation of an aversive US). It is also possible that higher test session activity levels interfered with the expression of a conditioned place aversion in the ethanol-preexposed mice. However, given the general similarity between their activity level and that of mice showing robust place aversion in other studies (e.g., Experiment 1), the lack of place aversion in the ethanol-preexposed mice is more likely due to failure to establish a CS-ethanol association.

\section{EXPERIMENT 3}

One interpretation of Experiments 1 and 2 is that mice resolve the potential ambiguity of the mid-CS injection procedure by learning only the first of the two contingencies embedded within that procedure-that is, the postCS ethanol contingency that normally produces conditioned place aversion. In Experiment 3, we examined another potentially ambiguous contingency. More specifically, the experimental mice received an injection of ethanol both before and after each presentation of the CS + (Figure 1B). Thus, as in Experiment 1, mice were exposed on each trial to a contingency that would be expected to produce place preference (ethanol before CS) and to a contingency that would be expected to produce place aversion (ethanol after CS). However, unlike in Experiment 1, the first contingency experienced on each trial was the one that was normally expected to produce conditioned place preference.

\section{Method}

This experiment began with a habituation session, in which the mice were weighed, injected with saline $(12.5 \mathrm{ml} / \mathrm{kg})$, and placed in the apparatus on a smooth paper floor. After $5 \mathrm{~min}$, they were removed, injected again with saline, and returned to the home cage. The mice were then randomly assigned to one of three large place conditioning treatment groups: before, after, or both $(n=29-32$ per group). The mice within each of these groups were randomly assigned to either grid + or grid - conditioning subgroups $(n=14-16$ per subgroup) and were exposed to a series of Pavlovian conditioning trials beginning $72 \mathrm{~h}$ after the habituation session. Each trial lasted $5 \mathrm{~min}$. The mice in the before group were injected with ethanol $(2 \mathrm{~g} / \mathrm{kg})$ immediately before placement on their assigned floor, whereas the mice in the after group received ethanol immediately after removal from the floor. The mice in the both group received an ethanol $(2-\mathrm{g} / \mathrm{kg})$ injection immediately before and after each exposure to the CS + floor. To match groups for the number of injections on each trial, the mice in the before group were injected with saline upon removal from the apparatus, and the mice in the after group were injected with saline immediately before each trial. The order of $\mathrm{CS}+$ and $\mathrm{CS}-$ trials was counterbalanced within each conditioning subgroup.

On the day after each mouse had received two conditioning trials of each type, all the mice received a 60-min floor preference test identical to that described in the previous experiments. After a 72-h 
break, all the mice received an additional two conditioning trials of each type followed by a second 60 -min preference test.

\section{Results}

Owing to equipment problems, test data from one mouse (after group) were removed from the analysis. Group sizes are indicated in the caption for Figure 4.

Figure 4 depicts results of the first preference test (left panel), conducted after the first two conditioning trials, and the second preference test (right panel), conducted after all four conditioning trials. As expected, the before group showed a conditioned preference. In contrast, the after group showed a weak trend toward conditioned aversion. Of greatest interest, however, the both group, which received an ethanol injection both before and after each CS + exposure, showed a conditioned preference greater than that shown by the before group.

The foregoing observations were supported by the outcome of a three-way repeated measures ANOVA (group $\times$ conditioning subgroup $\times$ test) that yielded a significant main effect of conditioning subgroup $[F(1,86)=18.5$, $p<.0001]$ and a significant group $\times$ conditioning subgroup interaction $[F(2,86)=14.9, p<.0001]$. There were no significant effects involving test. Follow-up comparisons (collapsed across tests) showed that the difference between the grid + and the grid - subgroups was significant within both the before and the both groups (Bonferronicorrected $p \mathrm{~s}<.03$ ), confirming the development of conditioned place preference. However, the difference between the grid + and the grid - subgroups was not significant for the after group, indicating an absence of conditioning. An additional three-way ANOVA was conducted without the after group's data to determine whether the other two groups differed in magnitude of conditioned preference. This analysis showed a significant group $\times$ conditioning subgroup interaction $[F(1,57)=5.6, p<.03]$, reflecting the development of stronger conditioned preference in the both group than in the before group.

Mean test activity rates (collapsed across tests) were $33.3 \pm 1.1,28.0 \pm 1.2$, and $31.1 \pm 1.3$ counts per minute for the before, both, and after groups, respectively. A twoway (group $\times$ test) ANOVA yielded a significant main effect of group $[F(2,89)=5.3, p<.01]$. Follow-up comparisons indicated a significant difference between the before and both groups (Bonferroni-corrected $p<.01$ ) but not between any other pair of groups. This analysis also showed a significant main effect of test $[F(1,89)=$ $9.9, p<.005]$, reflecting a small decrease in overall activity rate between the first $(32.0 \pm 0.8)$ and second $(29.6 \pm 0.08)$ tests. There was no group $\times$ test interaction. Although reduced test session activity in the both group may have enhanced its expression of conditioned place preference, a similar-sized difference in activity between the first and second tests was not accompanied by an increase in place preference across tests.

\section{Discussion}

The both group, which was exposed on each trial to contingencies that have previously been shown separately to produce strong conditioned preference and strong con-

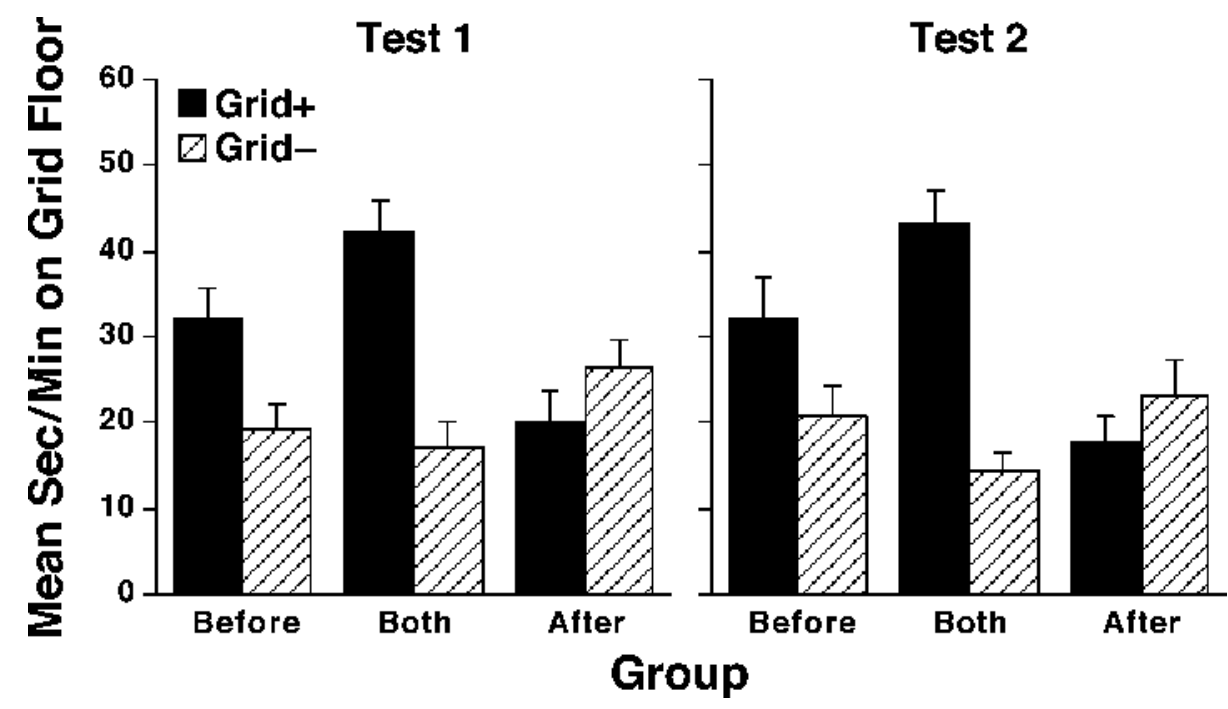

Figure 4. Mean seconds per minute $(+S E M)$ spent on the grid floor during the two 60-min floor preference test sessions in Experiment 3. The first test (left panel) was given after each mouse had received two conditioning trials of each type, whereas the second test (right panel) occurred after each mouse had received four trials of each type. The mice in the both group received injections of ethanol $(2 \mathrm{~g} / \mathrm{kg})$ both before and after each 5 -min conditioned stimulus $(\mathrm{CS}+)$ exposure. The mice in the before group received an ethanol injection before (and a saline injection after) each CS+ exposure, whereas the after group received an ethanol injection after (and a saline injection before) each CS+ exposure. Grid+ and grid - refer to the counterbalanced conditioning subgroups that received the grid floor paired or unpaired with ethanol injection, respectively $(n=14-16$ per subgroup). 
ditioned aversion, showed conditioned preference. Thus, the outcome is generally consistent with Experiment 1 in showing a primacy effect - that is, dominance by the first contingency experienced on each trial rather than an interaction between oppositely valenced unconditioned ethanol effects.

One might argue that ethanol's positive motivationaleffect predominated in the both group because the experimental contingency actually included a proximal US preexposure manipulation. That is, independently of its temporal relationship to the CS, injection of ethanol $5 \mathrm{~min}$ before the post-CS ethanol injection might have interfered with the ability of that post-CS injection to evoke an aversive response, an effect previously reported when proximal ethanol preexposure injections were given $1 \mathrm{~h}$ before postCS injections (Cunningham, Tull, et al., 2002). However, the fact that the conditioned preference shown by the both group was significantly larger than that seen in the before group indicates that these results cannot be explained simply in terms of a reduction in ethanol's aversive effects. One possibility is that the mice might have processed the second (post-CS) ethanol injection as a continuation of the first ethanol injection. This suggestion is supported by the observation that the effect of two closely spaced 2 $\mathrm{g} / \mathrm{kg}$ injections (one pre-CS and one post-CS) was quite similar to what one would expect from a single large (4$\mathrm{g} / \mathrm{kg}$ ) pre-CS ethanol injection.

The unexpected absence of significant conditioned aversion in the after group also raises the possibility that other factors might have influenced the outcome of Experiment 3. Given many previous demonstrations of robust conditioned place aversion produced by post-CS ethanol injections (e.g., Cunningham \& Henderson, 2000; Cunningham et al., 1998; Cunningham et al., 1997; Cunningham, Tull, et al., 2002), including the results of Experiments 1 and 2, it seems possible that pre-CS injection of saline may have interfered with acquisition of conditioned place aversion in the after group in Experiment 3. This interference could have been due either to CS properties of the pretrial saline injection (e.g., handling/injection cues might have overshadowed the floor CS) or to the ability of that pretrial saline injection to somehow reduce the aversive response to the posttrial ethanol injection (e.g., handling/ injection stress might have functioned as a generalized form of proximal US preexposure). In the latter case, however, one must argue that the close proximity of the saline pretrial injection was critical, because previous studies have shown that saline injections given in the home cage $1 \mathrm{~h}$ before post-CS ethanol injections do not prevent acquisition of conditioned place aversion (Cunningham, Tull, et al., 2002).

The effect of ethanol preexposure on place conditioning produced by the both procedure was not evaluated, for two reasons. First, repeated exposure to the cumulative dose that would have been produced by series of three $2-\mathrm{g} / \mathrm{kg}$ injections at 1-h intervals (proximal preexposure procedure) would likely have compromised the health and survival of the animals. Second, the finding that a post-CS in- jection appeared to enhance (rather than reduce) the place preference produced by the pre-CS injection already suggested that the mice in the both group experienced only a rewarding effect and not an aversive effect of ethanol, thus reducing interest in a manipulation that selectively interfered with ethanol's aversive effects.

\section{GENERAL DISCUSSION}

In these experiments, mice were exposed on every CS+ trial to contingencies that, when given separately, produced either conditioned place preference or conditioned place aversion. Although one might have expected joint application of these contingencies to produce interference, the subjects generally behaved as if they had been exposed only to the contingency that occurred first on each trial. Thus, the mice given mid-CS ethanol injections (Figure 1A) developed a conditioned place aversion, just like the mice that received only post-CS injections (Experiment 1). Moreover, the mice given both pre- and post-CS injections (Figure 1B) developed conditioned place preference, an outcome in the same direction as that shown by the mice given only pre-CS injections (Experiment 3 ). The outcome of Experiment 2, which showed no evidence of conditioned place preference despite exposure to a manipulation (ethanol preexposure) that eliminated conditioned place aversion, suggests that the mice given the mid-CS procedure were completely insensitive to the contingency that normally produces a strong conditioned place preference (i.e., injection of ethanol immediately before 5-min of exposure to the CS).

It is possible that the apparent failure to process the second contingency embedded within both of these "ambiguous" procedures was dependent on the pairing of only a single CS with ethanol in each procedure. That is, use of a single CS in these procedures may "force" subjects to process the ethanol US in only one way (rewarding or aversive), depending on whether ethanol or the CS occurs first. This analysis does not necessarily imply that mice experience only the rewarding effect or only the aversive effect. Rather, they might be incapable of associating a given CS with more than one motivational effect. In this regard, it would be interesting to know, for example, whether conditioned place preference would develop in a midtrial procedure if different CSs were presented before and after ethanol injection on the same conditioning trial. It is possible that the same ethanol injection could produce both conditioned aversion to the first CS and conditioned preference for the second CS.

The results from Experiment 1 lend further support to the interpretation offered for a recent series of ethanol place conditioning studies involving intragastric (i.g.) infusion of ethanol (Cunningham, Clemans, \& Fidler, 2002). These studies showed that, in contrast to i.p. injection, i.g. infusion of ethanol (through a catheter surgically implanted in the stomach) immediately before CS exposure unexpectedly produced conditioned place aversion. This outcome was explained by suggesting that administration by the i.g. 
route produced a delay in the onset of ethanol's aversive effect in such a way that the subjects did not fully experience this effect until after they had been in the apparatus for a minute or two. In other words, the authors proposed that a pretrial i.g. infusion of ethanol was functionally similar to a midtrial i.p. injection of ethanol. This hypothesis was supported by showing that the introduction of a short (5-min) delay between the i.g. infusion and the exposure to the CS + was sufficient to allow the aversive effect of ethanol to be elicited and to dissipate, resulting in the acquisition of conditioned place preference (Cunningham, Clemens, \& Fidler, 2002). Presumably, the time course of this process is much more rapid when i.p. injections are given, because there is no evidence for development of conditioned place aversion in mice when ethanol is injected i.p. immediately before CS exposure.

This analysis of the i.g. infusion studies illustrates the importance of attending carefully to the temporal relationship between the CS and the ethanol effects in place conditioning studies. Although it has been convenienthere to focus on only two general categories of contingencies (ethanol before CS and ethanol after CS), one should not assume that pre-CS contingency is the only relationship that will produce conditioned place preference or that post-CS contingency will invariably produce conditioned place aversion. In future studies, a broader range of temporal variations must be examined, especially in the "ambiguous" procedures studied here. It will also be important to examine other abused drugs to address the generality of the findings observed here.

In general, our interpretation of the effect of pre- and post-CS ethanol exposure is most consistent with conditioning theories that conceptualize USs as events that can activate multiple, independentrepresentational components (e.g., Wagner \& Brandon, 1989). The injection (or infusion) of ethanol is hypothesized to produce an initial, short-lived aversive reaction related to the novelty of the onset of intoxication, followed by a longer lasting positive motivational response. Although these motivational responses occur sequentially, they do not appear to be linked mechanistically in the manner suggested by opponent process theory (Solomon, 1977). The principal evidence against that possibility is the finding that distal or proximal preexposure to ethanol interferes with the development of conditioned place aversion but has no apparent effect on the development of conditioned place preference (Cunningham, Tull, et al., 2002). The present findings add to our understanding of these motivationally opposite effects by showing that when the conditions of learning provide the opportunity for a single CS to be associated with both effects, behavior is determined by the first effect that occurs after CS onset.

\section{REFERENCES}

Bardo, M. T., \& Neisewander, J. L. (1986). Single-trial conditioned place preference using intravenous morphine. Pharmacology, Biochemistry \& Behavior, 25, 1101-1105.
Cunningham, C. L. (1993). Pavlovian drug conditioning. In F. van Haaren (Ed.), Methods in behavioral pharmacology (pp. 349-381). Amsterdam: Elsevier.

Cunningham, C. L. (1995). Localization of genes influencing ethanolinduced conditioned place preference and locomotor activity in BXD recombinant inbred mice. Psychopharmacology, 120, 28-41.

Cunningham, C. L., Clemans, J. M., \& Fidler, T. L. (2002). Injection timing determines whether intragastric ethanol produces conditioned place preference or aversion in mice. Pharmacology, Biochemistry \& Behavior, 72, 659-668.

Cunningham, C. L., Dickinson, S. D., \& OKorn, D. M. (1995). Naloxone facilitates extinction but does not affect acquisition or expression of ethanol-induced conditioned place preference. Experimental \& Clinical Psychopharmacology, 3, 330-343.

Cunningham, C. L., \& Henderson, C. M. (2000). Ethanol-induced conditioned place aversion in mice. Behavioural Pharmacology, 11, 591-602.

Cunningham, C. L., Henderson, C. M., \& Bormann, N. M. (1998). Extinction of ethanol-induced conditioned place preference and conditioned place aversion: Effects of naloxone. Psychopharmacology, 139, 62-70.

Cunningham, C. L., Niehus, D. R., Malott, D. H., \& Prather, L. K. (1992). Genetic differences in the rewarding and activating effects of morphine and ethanol. Psychopharmacology, 107, 385-393.

Cunningham, C. L., Okorn, D. M., \& Howard, C. E. (1997). Interstimulus interval determines whether ethanol produces conditioned place preference or aversion in mice. Animal Learning \& Behavior, 25, 31-42.

Cunningham, C. L., Tull, L. E., Rindal, K. E., \& Meyer, P. J. (2002). Distal and proximal pre-exposure to ethanol in the place conditioning task: Tolerance to aversive effect, sensitization to activating effect, but no change in rewarding effect. Psychopharmacology, 160, 414-424.

Fudala, P. J., \& Iwamoto, E. T. (1987). Conditioned aversion after delay place conditioning with nicotine. Psychopharmacology, 92, 376-381.

Fudala, P. J., \& Iwamoto, E. T. (1990). Conditioned aversion after delay place conditioning with amphetamine. Pharmacology, Biochemistry \& Behavior, 35, 89-92.

Jorenby, D. E., Steinpreis, R. E., Sherman, J. E., \& Baker, T. B. (1990). Aversion instead of preference learning indicated by nicotine place conditioning in rats. Psychopharmacology, 101, 533-538.

Reicher, M. A., \& Holman, E. W. (1977). Location preference and flavor aversion reinforced by amphetamine in rats. Animal Learning \& Behavior, 5, 343-346.

Rescorla, R. A., \& Solomon, R. L. (1967). Two-process learning theory: Relationships between Pavlovian conditioning and instrumental learning. Psychological Review, 74, 151-182.

Risinger, F. O., \& OAKES, R. A. (1995). Nicotine-induced conditioned place preference and conditioned place aversion in mice. Pharmacology, Biochemistry \& Behavior, 51, 457-461.

Solomon, R. L. (1977). An opponent-process theory of acquired motivation: The affective dynamics of addiction. In M. E. P. Seligman \& J. D. Maser (Eds.), Psychopathology: Experimental models (pp. 66103). San Francisco: W. H. Freeman.

Wagner, A. R., \& Brandon, S. E. (1989). Evolution of a structured connectionist model of Pavlovian conditioning (AESOP). In S. B. Klein \& R. R. Mowrer (Eds.), Contemporary learning theories: Pavlovian conditioning and the status of traditional learning theory (pp. 149-189). Hillsdale, NJ: Erlbaum.

Wall, A.-M., Hinson, R. E., Schmidt, E., Johnston, C., \& StreaTHER, A. (1990). Place conditioning with d-amphetamine: The effect of the CS-UCS interval and evidence of a place avoidance. Animal Learning \& Behavior, 18, 393-400.

(Manuscript received January 31, 2003; revision accepted for publication May 28, 2003.) 